\title{
Clinical Presentation of Obstructive Sleep Apnea in Patients with Chronic Kidney Disease
}

\author{
David D. M. Nicholl, B.H.Sc. ${ }^{\text {; }}$ Sofia B. Ahmed, M.D., M.M.Sc. ${ }^{1,2}$; Andrea H. S. Loewen, M.D.1,3; Brenda R. Hemmelgarn, M.D., Ph.D. ${ }^{1,2}$;
} Darlene Y. Sola, B.Sc.N. '; Jaime M. Beecroft, M.Sc.3; Tanvir C. Turin, M.B.B.S., Ph.D. 1; Patrick J. Hanly, M.D.1,3

${ }^{1}$ Department of Medicine, Faculty of Medicine, University of Calgary, Calgary, Alberta, Canada; ${ }^{2}$ Alberta Kidney Disease Network, Alberta, Canada; ${ }^{3}$ Sleep Centre, Foothills Medical Centre, University of Calgary, Calgary, Alberta, Canada

Background: Obstructive sleep apnea (OSA) is an important and common comorbidity in patients with chronic kidney disease (CKD). However, few studies have addressed how OSA presents in this patient population and whether it is clinically apparent.

Objective: The objectives of this study were to determine if the prevalence and severity of sleep related symptoms distinguished CKD patients with OSA from those without apnea, and whether the clinical presentation of OSA in CKD patients differed from the general OSA population.

Methods: One hundred nineteen patients were recruited from outpatient nephrology clinics. All patients completed a sleep history questionnaire, the Epworth Sleepiness Scale (daytime sleepiness, ESS > 10), the Pittsburgh Sleep Quality Index (poor sleep quality, PSQI > 5), and underwent overnight cardiopulmonary monitoring for determination of sleep apnea (respiratory disturbance index $\geq 15$ ). CKD patients with OSA ( $n=46$ ) were compared to (1) CKD patients without OSA $(n=73)$ and $(2)$ OSA patients without CKD $(n=230)$ who were referred to the sleep centre.

Results: The prevalence of OSA symptoms and PSQI scores did not differ between CKD patients with OSA and CKD patients without apnea. Although the prevalence of daytime sleepiness was higher in CKD patients with OSA compared to CKD patients without apnea ( $39 \%$ vs. $19 \%, p=0.033$ ), both daytime sleepiness and other symptoms of sleep apnea were considerably less frequent than in OSA patients without a history of kidney disease.

Conclusions: The presence of OSA in patients with CKD is unlikely to be clinically apparent. Consequently, objective cardiopulmonary monitoring during sleep is required to reliably identify this comorbidity.

Keywords: Obstructive sleep apnea, chronic kidney disease, snoring, symptoms, daytime sleepiness

Citation: Nicholl DDM; Ahmed SB; Loewen AHS; Hemmelgarn BR; Sola DY; Beecroft JM; Turin TC; Hanly PJ. Clinical presentation of obstructive sleep apnea in patients with chronic kidney disease. J Clin Sleep Med 2012;8(4):381-387.
There is growing evidence that obstructive sleep apnea 1 (OSA) is common in patients with chronic kidney disease who do not require chronic dialysis (CKD). ${ }^{1-7}$ The reported prevalence of OSA in this population has ranged from $27 \%$ to $54 \%,{ }^{2,3,5-7}$ which is considerably higher than the general population. ${ }^{8}$ The coexistence of OSA in patients with CKD is likely to have clinical relevance. In addition to causing impairment of sleep quality and daytime function, ${ }^{9}$ OSA increases the risk of systemic hypertension ${ }^{10}$ and vascular disease, ${ }^{11,12}$ both of which are common complications of CKD. ${ }^{13}$ Furthermore, OSA may accelerate the deterioration of kidney function in patients with CKD either directly, through the effect of hypoxia on the kidney, ${ }^{14-16}$ or indirectly, by increasing systemic blood pressure, inflammatory cytokines, and sympathetic nervous system activity. ${ }^{17-20}$ Since OSA can be effectively treated in many patients with continuous positive airway pressure therapy (CPAP), ${ }^{21}$ it is important that the disorder be recognized and formally diagnosed in this patient population.

In conventional sleep medicine practice, the investigation of OSA is usually prompted by a constellation of sleep related symptoms such as snoring, witnessed apneas during sleep, and daytime sleepiness. ${ }^{22}$ It is not clear whether this clinical presentation can reliably distinguish patients with and without OSA in

\section{BRIEF SUMMARY \\ Current Knowledge/Study Rationale: Although obstructive sleep ap- nea (OSA) is common in patients with chronic kidney disease (CKD), it may not be clinically apparent. Consequently, we compared the preva- lence and severity of sleep related symptoms in CKD patients with and without OSA and contrasted that with the clinical presentation of OSA in patients without CKD. \\ Study Impact: OSA is not likely to be clinically apparent in patients with CKD. Objective cardiopulmonary monitoring is required to reliably iden- tify OSA in this patient population.}

the CKD population. Few studies have investigated the clinical presentation of OSA in non-dialysis-dependent CKD. ${ }^{2,5}$ These studies have reported a lower prevalence of daytime sleepiness $^{2,5}$ and snoring ${ }^{2}$ in the CKD population, but have not distinguished CKD patients with OSA from those without apnea. Thus, it is unclear whether OSA is clinically apparent in this patient population.

The objectives of this study were to determine (1) if the prevalence and severity of sleep related symptoms distinguished CKD patients with OSA from those without apnea, and (2) if the clinical presentation of OSA in CKD patients differed from that in the general sleep apnea population. We 
addressed these objectives by describing the clinical sleep profile of CKD patients with OSA and comparing this to CKD patients without OSA and to OSA patients without a history of kidney disease.

\section{METHODS}

\section{Patient Recruitment}

Adult patients ( $\geq 18$ years) with CKD (as defined by an estimated glomerular filtration rate $[\mathrm{eGFR}]<60 \mathrm{~mL} / \mathrm{min} / 1.73 \mathrm{~m}^{2}$ according to the National Kidney Foundation Staging System $^{23}$ ) attending outpatient nephrology clinics were invited to participate in the study. eGFR at the time of the study visit was calculated using the Chronic Kidney Disease Epidemiology Collaboration (CKD-EPI) equation. ${ }^{24}$ Exclusion criteria included current treatment with supplemental oxygen, tracheostomy, and inability to give informed consent. Patients currently treated with CPAP therapy were included in the study if their original diagnostic sleep study and sleep questionnaire were available for review and their eGFR at the time of OSA diagnosis was known. A control group of OSA patients without a history of kidney disease, but similar OSA severity (based on the respiratory disturbance index [RDI]), who were referred to the Foothills Sleep Centre for suspected sleep apnea during the same time period were randomly selected from the clinical database. Selection was performed while blinded to other data from nocturnal cardiopulmonary monitoring, patient demographics, and symptoms. The study was approved by the University of Calgary Conjoint Health Research Ethics Board. Informed consent was obtained from all participants in accordance with the Declaration of Helsinki.

One hundred twenty-four CKD patients were recruited. Fifty-one met our criteria for a diagnosis of sleep apnea (RDI $\geq$ 15). Eight patients currently treated with CPAP were included in the study. Five patients with Cheyne-Stokes respiration (CSR) were excluded from further analyses. The remaining 46 CKD patients with OSA were first compared to the 73 CKD patients without OSA and then compared to 230 OSA patients without a history of kidney disease.

\section{Nocturnal Cardiopulmonary Monitoring}

Patients performed an unattended, overnight cardiopulmonary monitoring study at home (Remmers Sleep Recorder Model 4.2, Saga Tech Electronic, Calgary, AB, Canada). The monitor consists of an oximeter to record oxyhemoglobin saturation $\left(\mathrm{SaO}_{2}\right)$ and heart rate variability, a pressure transducer to record nasal airflow, a microphone to record snoring, and a body position sensor. The oximeter provides the data for an automated scoring algorithm, which calculates the RDI based on the number of episodes of oxyhemoglobin desaturation $\geq 4 \%$ per hour of monitoring. Nocturnal oxygen saturation was sampled at $1 \mathrm{~Hz}$. The Remmers Sleep Recorder has been validated by comparison to attended polysomnography. ${ }^{25,26}$ We defined sleep apnea as an RDI $\geq 15$ as this reflects moderate to severe sleep apnea which is likely to be clinically significant. ${ }^{25,26}$ The Remmers Sleep Recorder has a sensitivity of $98 \%$ and specificity of $88 \%$ for a designation criteria of RDI $\geq 15 .{ }^{26}$ The raw data were reviewed by a sleep medicine physician $(\mathrm{PJH})$, blinded to patients' kidney function and symptoms, who confirmed that the estimated RDI was accurate and determined whether apnea was central (CSR) or obstructive (OSA), based on the morphology of the airflow recordings. Nasal pressure recordings with a characteristic crescendo/decrescendo pattern and no evidence of airflow limitation were classified as CSR, whereas recordings without a crescendo/decrescendo pattern and with airflow limitation were classified as OSA.

\section{Subjective Measurements of Sleep Quality}

\section{Sleep History Questionnaire}

All patients completed a standardized sleep history questionnaire developed at Foothills Sleep Centre, which included a history of snoring, witnessed apnea during sleep and nocturnal choking, unrefreshing sleep, morning headaches, and memory impairment. Additionally, the questionnaire surveyed demographic information and medical history, including a history of obesity (body mass index $[\mathrm{BMI}] \geq 30 \mathrm{~kg} /$ $\mathrm{m}^{2}$ ), hypertension, cardiovascular disease (angina, myocardial infarction, coronary artery bypass surgery, or congestive heart failure), cerebrovascular disease (stroke or transient ischemic attack), diabetes, chronic obstructive pulmonary disease (COPD), and medications.

\section{Daytime Sleepiness}

All patients completed the Epworth Sleepiness Scale (ESS). ${ }^{27}$ The ESS is a self-administered questionnaire designed to measure the general level of daytime sleepiness. Patients rate on a scale of 0-3 how likely they are to fall asleep in 8 different situations that are commonly encountered. Total ESS scores range from $0-24$, with higher scores indicating more subjective daytime sleepiness. Specifically, an ESS score $>10$ is considered indicative of subjective daytime sleepiness. ${ }^{27}$

\section{Sleep Quality}

All CKD patients completed the Pittsburgh Sleep Quality Index (PSQI). ${ }^{28}$ The PSQI is a self-rated questionnaire that assesses sleep quality and disturbances over a 1-month time interval. Nineteen individual items generate 7 "component" scores: subjective sleep quality, sleep latency, sleep duration, habitual sleep efficiency, sleep disturbances, use of sleep medications, and daytime dysfunction. The sum of the seven component scores yields one global score, which ranges from 0-21. Higher scores indicate worse sleep quality, and PSQI global scores > 5 are considered indicative of poor sleep quality. ${ }^{28}$ PSQI data were not available for OSA patients without a history of kidney disease. All questionnaires were completed on the evening of overnight cardiopulmonary monitoring.

\section{Analysis}

Data are presented as mean \pm standard deviation or number (percentage). CKD patients with OSA were initially compared to CKD patients without OSA, and secondly to OSA patients without a history of kidney disease. The unpaired $t$-test or the Mann-Whitney U-test was used for comparisons between continuous variables while the $\chi^{2}$ test with Fischer exact test was used for dichotomous variables. Univariate and multivariate logistic regression models were used to identify 
Table 1-Characteristics of chronic kidney disease patients, stratified by OSA status

\begin{tabular}{|c|c|c|c|c|}
\hline & \multirow[t]{2}{*}{ All Patients } & \multicolumn{3}{|c|}{ OSA status } \\
\hline & & OSA & No Apnea & p-value \\
\hline N & 119 & 46 & 73 & - \\
\hline \multicolumn{5}{|l|}{ Nocturnal monitoring } \\
\hline $\mathrm{RDI}, / \mathrm{h}$ & $21.1 \pm 25.1$ & $43.3 \pm 28.4$ & $7.2 \pm 3.8$ & $<0.001$ \\
\hline Mean $\mathrm{SaO}_{2}, \%$ & $91.5 \pm 3.2$ & $89.6 \pm 3.3$ & $92.7 \pm 2.4$ & $<0.001$ \\
\hline $\mathrm{SaO}_{2}<90 \%, \%$ monitoring time & $22.6 \pm 28.3$ & $39.5 \pm 30.3$ & $11.9 \pm 20.9$ & $<0.001$ \\
\hline Total monitoring time, $\mathrm{h}$ & $7.2 \pm 1.5$ & $7.1 \pm 1.7$ & $7.3 \pm 1.4$ & 0.641 \\
\hline Reported sleep time, \% monitoring time & $81 \pm 15$ & $80 \pm 16$ & $81 \pm 14$ & 0.686 \\
\hline \multicolumn{5}{|l|}{ Demographics } \\
\hline Age, years & $65 \pm 12$ & $67 \pm 10$ & $64 \pm 13$ & 0.277 \\
\hline Male & $75(63)$ & $34(74)$ & $41(56)$ & 0.055 \\
\hline $\mathrm{BMl}, \mathrm{kg} / \mathrm{m}^{2}$ & $31.2 \pm 8.3$ & $34.0 \pm 9.6$ & $29.5 \pm 6.8$ & 0.004 \\
\hline \multicolumn{5}{|l|}{ Comorbidities } \\
\hline Obesity & $56(47)$ & $27(59)$ & $29(40)$ & 0.057 \\
\hline Hypertension & $108(91)$ & $42(91)$ & $66(90)$ & 1.000 \\
\hline Cardiovascular disease & $24(20)$ & $14(30)$ & $10(14)$ & 0.035 \\
\hline Cerebrovascular disease & $8(7)$ & $3(7)$ & $5(7)$ & 1.000 \\
\hline Diabetes & $47(39)$ & $21(46)$ & $26(36)$ & 0.337 \\
\hline COPD & $6(5)$ & $3(7)$ & $3(4)$ & 0.675 \\
\hline \multicolumn{5}{|l|}{ Medications } \\
\hline Sedatives & $10(8)$ & $3(7)$ & $7(10)$ & 0.739 \\
\hline Antidepressants & $5(4)$ & $3(7)$ & $2(3)$ & 0.373 \\
\hline
\end{tabular}

Data are mean $\pm \mathrm{SD}$ or number (percentage) of patients within group. OSA, obstructive sleep apnea; $\mathrm{RDI}$, respiratory disturbance index; $\mathrm{SaO}_{2}$, oxyhemoglobin saturation; BMI, body mass index; COPD, chronic obstructive pulmonary disease. *OSA versus No Apnea.

factors associated with OSA in CKD patients. Age, male gender, comorbidities (obesity, hypertension, cardiovascular disease, cerebrovascular disease, and diabetes), medications, and sleep related symptoms were included in the model. All model assumptions were tested and met. All statistical analyses were 2-sided and performed with SPSS V.17.0 (SPSS, Chicago, IL, USA). P-values $<0.05$ were considered statistically significant.

\section{RESULTS}

\section{Chronic Kidney Disease: OSA versus No Apnea}

\section{Patient Characteristics}

The nocturnal cardiopulmonary monitoring findings and clinical profile of CKD patients with and without OSA are shown in Table 1. By definition, the RDI was higher in CKD patients with OSA than in patients without sleep apnea. As expected, the severity of associated nocturnal hypoxemia was greater in CKD patients with OSA. Although CKD patients with OSA had a higher prevalence of cardiovascular disease than those without sleep apnea, they did not differ from non-apneic patients in terms of gender, age, other comorbidities, or medication use.

\section{Sleep Related Symptoms}

Sleep related symptoms for all CKD patients are displayed in Table 2. There were no differences in the prevalence of re- ported snoring, witnessed apnea, nocturnal choking, unrefreshing sleep, morning headaches, or memory impairment between CKD patients with and without OSA. Although the mean ESS was not different between groups, the proportion of patients with an abnormal ESS score (ESS > 10) was greater in CKD patients with OSA ( $39 \%$ versus $19 \%, p=0.033)$. No differences were observed between mean PSQI global scores or the proportion of patients with an abnormal score (PSQI $>5$ ).

On univariate analysis (Table 3), only obesity, cardiovascular disease, and daytime sleepiness $(\mathrm{ESS}>10)$ were associated with OSA in CKD patents. Male gender and witnessed apneas during sleep were of borderline significance. On multivariate analysis, only male gender was significantly associated with OSA in CKD patients.

\section{Obstructive Sleep Apnea: CKD versus No History of Kidney Disease}

\section{Patient Characteristics}

The nocturnal cardiopulmonary monitoring findings and clinical profile of OSA patients with CKD compared to those without a history of kidney disease are shown in Table 4. By study design, the RDI was similar between the two groups. However, the severity of associated nocturnal hypoxemia was greater in OSA patients with CKD than in OSA patients without a history of kidney disease. OSA patients with CKD were older and a greater proportion had hypertension and diabetes. However, there were no intergroup differences in 
Table 2-Sleep-related symptoms of chronic kidney disease patients, stratified by OSA status

\begin{tabular}{|c|c|c|c|c|}
\hline & \multirow[t]{2}{*}{ All Patients } & \multicolumn{3}{|c|}{ OSA status } \\
\hline & & OSA & No Apnea & $p$-value* \\
\hline $\mathrm{N}$ & 119 & 46 & 73 & - \\
\hline Snoring, \% & $93(78)$ & $39(85)$ & $54(74)$ & 0.665 \\
\hline Witnessed apnea, \% & $30(25)$ & $16(35)$ & $14(19)$ & 0.082 \\
\hline Nocturnal choking, $\%$ & $34(29)$ & $17(37)$ & $17(23)$ & 0.145 \\
\hline Epworth Sleepiness Scale & $7.9 \pm 4.7$ & $8.8 \pm 5.2$ & $7.3 \pm 4.3$ & 0.087 \\
\hline Daytime sleepiness (ESS > 10), \% & $32(27)$ & $18(39)$ & $14(19)$ & 0.033 \\
\hline Pittsburgh Sleep Quality Index, Global Score & $6.0 \pm 3.9$ & $6.2 \pm 3.9$ & $5.9 \pm 4.0$ & 0.779 \\
\hline Poor sleep quality (PSQI > 5), \% & $52(44)$ & $21(46)$ & $31(42)$ & 0.707 \\
\hline Unrefreshing sleep, $\%$ & $50(42)$ & $19(41)$ & $31(42)$ & 1.000 \\
\hline Morning headaches, \% & $9(8)$ & $4(9)$ & $5(7)$ & 0.733 \\
\hline Memory impairment, \% & $52(43)$ & $23(50)$ & $29(40)$ & 0.343 \\
\hline
\end{tabular}

Data are mean \pm SD or number (percentage) of patients within group. OSA, obstructive sleep apnea; ESS, Epworth Sleepiness Scale; PSQI, Pittsburgh Sleep Quality Index. *OSA versus No Apnea.

Table 3-Predictors of obstructive sleep apnea in chronic kidney disease patients by univariate and multivariate analysis

\begin{tabular}{|c|c|c|c|c|c|c|}
\hline & \multicolumn{3}{|c|}{ Univariate } & \multicolumn{3}{|c|}{ Multivariate } \\
\hline & OR & $95 \% \mathrm{Cl}$ & P-value & OR & $95 \% \mathrm{Cl}$ & $\mathrm{p}$-value \\
\hline \multicolumn{7}{|l|}{ Demographics } \\
\hline Age, years & 1.02 & $0.99,1.05$ & 0.275 & 1.01 & $0.97,1.05$ & 0.686 \\
\hline Male & 2.21 & $0.99,4.94$ & 0.053 & 3.80 & $1.36,10.7$ & 0.011 \\
\hline Obesity & 2.17 & $1.02,4.65$ & 0.046 & 2.06 & $0.74,5.70$ & 0.165 \\
\hline Hypertension & 1.11 & $0.31,4.04$ & 0.870 & 0.84 & $0.18,4.00$ & 0.827 \\
\hline Cardiovascular disease & 2.76 & $1.10,6.89$ & 0.030 & 1.52 & $0.43,6.37$ & 0.512 \\
\hline Cerebrovascular disease & 0.95 & $0.22,4.17$ & 0.945 & 0.75 & $0.11,5.05$ & 0.766 \\
\hline Diabetes & 1.52 & $0.72,3.22$ & 0.277 & 1.11 & $0.41,3.03$ & 0.834 \\
\hline Sedatives & 0.66 & $0.16,2.68$ & 0.559 & 0.44 & $0.08,2.46$ & 0.350 \\
\hline Antidepressants & 2.48 & $0.40,15.4$ & 0.331 & 2.60 & $0.28,24.2$ & 0.401 \\
\hline \multicolumn{7}{|l|}{ Sleep Related Symptoms } \\
\hline Snoring & 1.27 & $0.53,3.04$ & 0.596 & 1.84 & $0.64,5.29$ & 0.255 \\
\hline Witnessed apnea & 2.25 & $0.97,5.21$ & 0.059 & 1.80 & $0.59,5.48$ & 0.298 \\
\hline Nocturnal choking & 1.93 & $0.86,4.33$ & 0.110 & 1.17 & $0.38,3.56$ & 0.787 \\
\hline Daytime sleepiness (ESS > 10) & 2.62 & $1.14,6.02$ & 0.023 & 1.85 & $0.66,5.15$ & 0.242 \\
\hline Poor sleep quality (PSQI > 5) & 1.16 & $0.55,2.45$ & 0.702 & 1.17 & $0.44,3.13$ & 0.751 \\
\hline Unrefreshing sleep & 0.95 & $0.45,2.02$ & 0.901 & 0.77 & $0.29,2.06$ & 0.605 \\
\hline Morning headaches & 1.30 & $0.33,5.10$ & 0.711 & 1.41 & $0.22,9.24$ & 0.719 \\
\hline Memory impairment & 1.52 & $0.72,3.19$ & 0.272 & 1.74 & $0.64,4.70$ & 0.276 \\
\hline
\end{tabular}

OR, odds ratio; Cl, confidence interval; ESS, Epworth Sleepiness Scale; PSQI, Pittsburgh Sleep Quality Index.

gender, BMI, medication use, or the prevalence of obesity or coexisting cardiovascular disease, cerebrovascular disease, and COPD.

\section{Sleep related Symptoms}

Snoring, witnessed apnea, unrefreshing sleep, and morning headaches were reported less often by OSA patients with CKD than OSA patients without a history of kidney disease (Table 5). In addition, the prevalence of daytime sleepiness was lower in OSA patients with CKD than in OSA patients without a history of kidney disease $(39 \%$ versus $63 \%, p=0.005)$, though this result may be partly explained by selection bias since OSA patients with CKD were not referred for evaluation of sleep complaints.

\section{DISCUSSION}

The presence of OSA in patients with CKD was associated with only one of the traditional risk factors for sleep apnea, namely male gender. The prevalence of sleep related symptoms was lower in OSA patients with CKD than OSA patients without a history of kidney disease. More importantly, sleep related symptoms did not distinguish CKD patients with OSA from CKD patients without OSA. 
Table 4-Characteristics of obstructive sleep apnea patients, stratified by chronic kidney disease status

\begin{tabular}{|c|c|c|c|}
\hline & CKD & No CKD & p-value* \\
\hline $\mathrm{N}$ & 46 & 230 & - \\
\hline \multicolumn{4}{|l|}{ Nocturnal monitoring } \\
\hline $\mathrm{RDI}, / \mathrm{h}$ & $43.3 \pm 28.4$ & $42.7 \pm 12.5$ & 0.888 \\
\hline Mean $\mathrm{SaO}_{2}, \%$ & $89.6 \pm 3.3$ & $90.6 \pm 2.7$ & 0.052 \\
\hline $\mathrm{SaO}_{2}<90, \%$ monitoring time & $39.5 \pm 30.3$ & $30.1 \pm 24.2$ & 0.048 \\
\hline Total monitoring time, $\mathrm{h}$ & $7.1 \pm 1.7$ & $7.0 \pm 12.3$ & 0.711 \\
\hline Reported sleep time, \% monitoring time & $80 \pm 16$ & $83 \pm 13$ & 0.268 \\
\hline \multicolumn{4}{|l|}{ Demographics } \\
\hline Age, years & $67 \pm 10$ & $51 \pm 10$ & $<0.001$ \\
\hline Male & $34(74)$ & $161(70)$ & 0.479 \\
\hline BMI, kg/m² & $34.0 \pm 9.6$ & $35.0 \pm 8.2$ & 0.544 \\
\hline \multicolumn{4}{|l|}{ Comorbidities } \\
\hline Obesity & $27(59)$ & $156(68)$ & 0.297 \\
\hline Hypertension & $42(91)$ & $129(56)$ & $<0.001$ \\
\hline Cardiovascular disease & $14(30)$ & $45(20)$ & 0.116 \\
\hline Cerebrovascular disease & $3(7)$ & $5(2)$ & 0.132 \\
\hline Diabetes & $21(46)$ & $26(11)$ & $<0.001$ \\
\hline COPD & $3(7)$ & $41(18)$ & 0.075 \\
\hline \multicolumn{4}{|l|}{ Medications } \\
\hline Sedatives & $3(7)$ & $9(4)$ & 0.428 \\
\hline Antidepressants & $3(7)$ & $26(11)$ & 0.436 \\
\hline
\end{tabular}

Data are mean $\pm \mathrm{SD}$ or number (percentage) of patients within group. $\mathrm{CKD}$, chronic kidney disease; $\mathrm{RDI}$, respiratory disturbance index; $\mathrm{SaO}_{2}$, oxyhemoglobin saturation BMI, body mass index; COPD, chronic obstructive pulmonary disease. ${ }^{*} \mathrm{CKD}$ versus No CKD.

Table 5-Sleep-related symptoms of obstructive sleep apnea patients, stratified by chronic kidney disease status

$\begin{array}{lccr} & \text { CKD } & \text { No CKD } & \text { p-value* } \\ \text { N } & 46 & 230 & - \\ \text { Snoring, \% } & 39(85) & 225(98) & <0.001 \\ \text { Witnessed apnea, \% } & 16(35) & 179(78) & <0.001 \\ \text { Nocturnal choking, \% } & 17(37) & 120(52) & 0.075 \\ \text { Epworth Sleepiness Scale } & 8.8 \pm 5.2 & 12.2 \pm 5.7 & <0.001 \\ \text { Daytime Sleepiness (ESS > 10), \% } & 18(39) & 144(63) & 0.005 \\ \text { Unrefreshing sleep, \% } & 19(41) & 192(83) & <0.001 \\ \text { Morning headaches, \% } & 4(9) & 86(37) & <0.001 \\ \text { Memory impairment, \% } & 23(50) & 143(62) & 0.248\end{array}$

Data are mean $\pm S D$ or number (percentage) of patients within group. CKD, chronic kidney disease; ESS, Epworth Sleepiness Scale. *CKD versus No CKD.

Only two previous studies have evaluated the clinical presentation of OSA in non-dialysis-dependent CKD. Markou et al. ${ }^{2}$ reported a low prevalence of excessive daytime sleepiness (ESS > 10) of $11.4 \%$, while the prevalence of snoring was found to be $40 \%$ in a cross-sectional study of 35 patients with CKD $\left(\mathrm{eGFR}=26.8 \mathrm{~mL} / \mathrm{min} / 1.73 \mathrm{~m}^{2}, 11-40\right)$. Their study was limited by a small sample size and they excluded patients with cardiovascular disease thereby limiting the generalizability of their findings as cardiovascular disease is a common comorbidity in this patient population..$^{13}$ Roumelioti et al. ${ }^{5}$ reported a higher prevalence of excessive daytime sleepiness (ESS $\geq 10$ ) of $29.3 \%$ in 89 CKD patients but used historical controls whose kidney function was undefined for comparison. Further, neither of these studies compared sleep related symptoms between CKD patients with OSA and nonapneic patients.

Our study addressed several of the limitations of these previous studies. First, we compared CKD patients with and without OSA. Second, our sample size was quite large, and all subjects were recruited from nephrology clinics, increasing the relevance of our findings to that patient population. Third, no inclusion or exclusion criteria were set with respect to age, gender, comorbidities, or medications, which improved the generalizability of our findings to the CKD population. In fact, our CKD population had a similar clinical profile to the Chronic Renal Insufficiency Cohort study. ${ }^{29}$

The lack of excessive daytime sleepiness in a significant proportion of OSA patients with CKD has been reported in other 
specific OSA populations, including those with stroke, ${ }^{30}$ heart failure, ${ }^{31}$ hypertension,,${ }^{32,33}$ and end-stage renal disease. ${ }^{34}$ There are a number of potential explanations for this observation that we can speculate on. First, it may reflect selection bias if the presence of sleep symptoms is not equally important in the recruitment of patients to the groups that are compared. Second, the complaint of subjective sleepiness may be overshadowed by other symptoms associated with chronic disease, such as anxiety or chronic fatigue, or side effects of their treatment such as medications. Third, the comorbid disease itself may hinder the development of excessive sleepiness through competing biologic mechanisms, such as augmented sympathetic activity in patients with chronic heart failure. Regardless of the explanation, the cumulative evidence indicates that daytime sleepiness is not a reliable diagnostic criterion for OSA in patients with many chronic medical disorders including CKD. Further, the absence of daytime sleepiness should not dissuade the clinician from considering a diagnosis of OSA in this patient population.

What are the clinical implications of our findings? OSA increases the risk of hypertension, ${ }^{10}$ cardiovascular, ${ }^{11}$ and cerebrovascular ${ }^{12}$ disease, all of which are important and highly prevalent complications of CKD. ${ }^{13}$ Further, OSA may also accelerate the deterioration of kidney function. ${ }^{14-20}$ As OSA can be effectively treated with CPAP therapy, ${ }^{21}$ it is important that this disorder be considered in this patient population and formally diagnosed. Male gender was the only significant predictor of OSA in our population of CKD patients. However, in conventional sleep medicine practice, the investigation of OSA is usually prompted by a constellation of sleep related symptoms including snoring, witnessed apneas during sleep, and daytime sleepiness, along with traditional risk factors for the disorder such as obesity and male gender. ${ }^{22}$ In our study, we found that we were unable to distinguish between CKD patients with OSA and CKD patients without OSA based solely on sleep related symptoms. Although the presentation of an obese male patient with CKD should prompt physicians to consider OSA, further clinical assessment for sleep apnea is unlikely to be helpful and objective cardio-pulmonary monitoring should be used to reliably diagnose the disorder.

Our study has limitations. First, the potential for selection bias exists as patients attending the nephrology clinics may have been more likely to participate if they suspected they had sleep apnea. We tried to limit the potential impact of this on our findings by emphasising that sleep related complaints were not required for recruitment. If such a bias did exist, it should have been reflected in a higher prevalence of sleep related symptoms in CKD patients with OSA which was not the case. Second, OSA patients with CKD were recruited differently than OSA patients with normal kidney function. Notwithstanding this difference, the primary purpose of describing the OSA group without kidney disease was to highlight the typical clinical stereotype of OSA, detected by the same methodology, and how infrequently CKD patients with OSA present in that way. Third, we did not objectively assess kidney function in OSA patients without a history of kidney disease. However, we were vigilant to ask all patients about kidney disease and excluded any whose history was suggestive of this.

Although male gender was the strongest predictor of OSA in patients with CKD, OSA is unlikely to be clinically apparent in this population. This is disconcerting, given the high prevalence of OSA in CKD and its potential impact on important clinical outcomes. ${ }^{1-7}$ Further studies are required to determine the validity and efficacy of OSA clinical prediction rules in patients with CKD. In the meantime, objective cardiopulmonary monitoring during sleep is required to reliably identify sleep apnea in this patient population.

\section{REFERENCES}

1. Kimmel PL, Miller G, Mendelson WB. Sleep apnea syndrome in chronic renal disease. Am J Med 1989;86:308-14.

2. Markou N, Kanakaki M, Myrianthefs $P$, et al. Sleep-disordered breathing in nondialyzed patients with chronic renal failure. Lung 2006;184:43-9.

3. Canales MT, Lui LY, Taylor BC, et al. Renal function and sleep-disordered breathing in older men. Nephrol Dial Transplant 2008;23:3908-14.

4. Sim JJ, Rasgon SA, Kujubu DA, et al. Sleep apnea in early and advanced chronic kidney disease: Kaiser Permanente Southern California cohort. Chest 2009;135:710-6.

5. Roumelioti ME, Buysse DJ, Sanders MH, Strollo P, Newman AB, Unruh ML. Sleep-disordered breathing and excessive daytime sleepiness in chronic kidney disease and hemodialysis. Clin J Am Soc Nephrol 2011;6:986-94.

6. Sakaguchi $\mathrm{Y}$, Shoji T, Kawabata H, et al. High prevalence of obstructive sleep apnea and its association with renal function among nondialysis chronic kidney disease patients in Japan: a cross-sectional study. Clin J Am Soc Nephrol $2011 ; 6: 995-1000$

7. Nicholl DD, Ahmed SB, Loewen AH, et al. Declining kidney function increases the prevalence of sleep apnea and nocturnal hypoxia. Chest 2012;141:1422-30.

8. Young T, Palta M, Dempsey J, Skatrud J, Weber S, Badr S. The occurrence of sleep-disordered breathing among middle-aged adults. N Engl J Med 1993;328:1230-5

9. Malhotra A, White DP. Obstructive sleep apnoea. Lancet 2002;360:237-45.

10. Peppard PE, Young T, Palta M, Skatrud J. Prospective study of the association between sleep-disordered breathing and hypertension. N Engl J Med 2000;342:1378-84.

11. Marin JM, Carrizo SJ, Vicente E, Agusti AG. Long-term cardiovascular outcomes in men with obstructive sleep apnoea-hypopnoea with or without treatment with continuous positive airway pressure: an observational study. Lancet 2005;365:1046-53.

12. Redline S, Yenokyan G, Gottlieb DJ, et al. Obstructive sleep apnea-hypopnea and incident stroke: the sleep heart health study. Am J Respir Crit Care Med 2010;182:269-77.

13. National Kidney Foundation. $K / D O Q$ I clinical practice guidelines for chronic kidney disease: evaluation, classification, and stratification. Am J Kidney Dis 2002;39:S1-266.

14. Fine LG, Norman JT. Chronic hypoxia as a mechanism of progression of chronic kidney diseases: from hypothesis to novel therapeutics. Kidney Int 2008;74:867-72.

15. Fine LG, Orphanides C, Norman JT. Progressive renal disease: the chronic hypoxia hypothesis. Kidney Int Suppl 1998;65:S74-8.

16. Ahmed SB, Ronksley PE, Hemmelgarn BR, et al. Nocturnal hypoxia and loss of kidney function. PLOS One 2011;6:e19029.

17. Zoccali $C$, Benedetto FA, Mallamaci $F$, et al. Left ventricular hypertrophy and nocturnal hypoxemia in hemodialysis patients. J Hypertens 2001;19:287-93.

18. Zoccali C, Benedetto FA, Tripepi G, et al. Nocturnal hypoxemia, night-day arterial pressure changes and left ventricular geometry in dialysis patients. Kidney Int 1998;53:1078-84.

19. Zoccali C, Mallamaci F, Tripepi G. Nocturnal hypoxemia predicts incident cardiovascular complications in dialysis patients. J Am Soc Nephrol 2002;13:729-33.

20. Zoccali C, Mallamaci F, Tripepi G, Benedetto FA. Autonomic neuropathy is linked to nocturnal hypoxaemia and to concentric hypertrophy and remodelling in dialysis patients. Nephrol Dial Transplant 2001;16:70-7.

21. Pressman MR, Benz RL, Schleifer CR, Peterson DD. Sleep disordered breathing in ESRD: acute beneficial effects of treatment with nasal continuous positive airway pressure. Kidney Int 1993;43:1134-9.

22. Sleep-related breathing disorders in adults: recommendations for syndrome definition and measurement techniques in clinical research. The Report of an American Academy of Sleep Medicine Task Force. Sleep 1999;22:667-89.

23. Levey AS, Coresh J, Balk E, et al. National Kidney Foundation practice guidelines for chronic kidney disease: evaluation, classification, and stratification. Ann Intern Med 2003;139:137-47. 
24. Levey AS, Stevens $L A$, Schmid $C H$, et al. A new equation to estimate glomerular filtration rate. Ann Intern Med 2009;150:604-12.

25. Issa FG, Morrison D, Hadjuk E, lyer A, Feroah T, Remmers JE. Digital monitoring of sleep-disordered breathing using snoring sound and arterial oxygen saturation. Am Rev Respir Dis 1993;148:1023-9.

26. Vazquez JC, Tsai WH, Flemons WW, et al. Automated analysis of digital oximetry in the diagnosis of obstructive sleep apnoea. Thorax 2000;55:302-7.

27. Johns MW. A new method for measuring daytime sleepiness: the Epworth sleepiness scale. Sleep 1991;14:540-5.

28. Buysse DJ, Reynolds CF 3rd, Monk TH, Berman SR, Kupfer DJ. The Pittsburgh Sleep Quality Index: a new instrument for psychiatric practice and research. Psychiatry Res 1989;28:193-213.

29. Lash JP, Go AS, Appel LJ, et al. Chronic Renal Insufficiency Cohort (CRIC) Study: baseline characteristics and associations with kidney function. Clin J Am Soc Nephrol 2009;4:1302-11.

30. Arzt M, Young T, Peppard PE, et al. Dissociation of obstructive sleep apnea from hypersomnolence and obesity in patients with stroke. Stroke 2010;41:e129-34.

31. Arzt M, Young T, Finn L, et al. Sleepiness and sleep in patients with both systolic heart failure and obstructive sleep apnea. Arch Intern Med 2006;166:1716-22.

32. Drager LF, Genta PR, Pedrosa RP, et al. Characteristics and predictors of obstructive sleep apnea in patients with systemic hypertension. Am J Cardiol 2010;105:1135-9.

33. Gus M, Goncalves SC, Martinez D, et al. Risk for obstructive sleep apnea by Berlin Questionnaire, but not daytime sleepiness, is associated with resistant hypertension: a case-control study. Am J Hypertens 2008;21:832-5.

34. Beecroft JM, Pierratos A, Hanly PJ. Clinical presentation of obstructive sleep apnea in patients with end-stage renal disease. J Clin Sleep Med 2009;5:115-21.

\section{ACKNOWLEDGMENTS}

The authors thank the Southern Alberta Renal Program for patient recruitment, the FMC Sleep Centre for sleep diagnostic testing and Ms. Patty Nielsen for her clerical assistance. This research was supported by Alberta Innovates-Health Solutions, O'Brien Centre, University of Calgary, and the Department of Medicine, University of Calgary.

\section{SUBMISSION \& CORRESPONDENCE INFORMATION}

Submitted for publication October, 2011

Submitted in final revised form January, 2012

Accepted for publication January, 2012

Address correspondence to: Patrick J. Hanly, M.D., 1421 Health Sciences Centre, 3330 Hospital Drive NW, Calgary, Alberta, Canada; Tel: (403) 210-8694; Fax: (403)

283-6151; E-mail: phanly@ucalgary.ca

\section{DISCLOSURE STATEMENT}

An abstract for this study was presented at the annual meeting of the American Society of Nephrology in Philadelphia, PA, November 8-13, 2011. This was not an industry supported study. The authors have indicated no financial conflicts of interest. 